\title{
Chiral dynamics of/with unstable particles
}

\author{
Evgeny Epelbaum ${ }^{1, \star}$, Hermann Krebs ${ }^{1, \star \star}$ and Ulf-G. Meißner ${ }^{2,3, \star \star \star}$ \\ ${ }^{1}$ Institut für Theoretische Physik II, Ruhr-Universität Bochum, D-44780 Bochum, Germany \\ ${ }^{2}$ Universität Bonn, Helmholtz Institut für Strahlen- und Kernphysik, Bethe Center for Theoretical Physics, D-53115 Bonn, Germany \\ ${ }^{3}$ Forschungszentrum Jülich, Institute für Kernphysik (IKP-3), Institute for Advanced Simulation (IAS-4) and Jülich Center for \\ Hadron Physics, D-52145 Jülich, Germany
}

\begin{abstract}
We review the achievements of the project C.9. Topics addressed include the magnetic moment of the $\rho$-meson, elastic pion-nucleon scattering in various settings of chiral perturbation theory, the reaction $\pi N \rightarrow \pi \pi N$ as well as new directions in chiral perturbation theory.
\end{abstract}

\section{Introduction}

The project $\mathbf{C . 9}$ has been funded over the third period of the SFB/TR 16. It grew out of the project C.3, that was funded in the first period. Within this project, we have carried out detailed investigations of pion-nucleon scattering using various formulations of baryon chiral perturbation theory (ChPT) and addressed the role of the $\Delta(1232)$ isobar. Furthermore, we have explored the possibility of extending the applicability of ChPT beyond the lowenergy region, verified the consistency of the complexmass renormalization scheme at the two-loop level and performed a number of applications in the two-nucleon sector. This project has been quite successful which is partly due to the intelligent use of the lump sum funds.

Our contribution is organized as follows. In section 2, we present the calculation of the pion-mass dependence of the magnetic moment of the $\rho$-meson. Sections 3 and 4 are devoted to elastic $\pi N$ scattering and the reaction $\pi N \rightarrow \pi \pi N$, respectively. Next, in section 5 , we discuss new directions in ChPT focusing, in particular, on the applicability of the complex-mass renormalization scheme beyond the one-loop level and a possibility to extend the ChPT framework beyond the low-energy region. Finally, section 6 describes the applications in the nucleon-nucleon (NN) sector. Here, we discuss the electromagnetic form factors of the deuteron, a non-perturbative inclusion of the subleading contact interaction in the ${ }^{1} \mathrm{~S}_{0}$ channel within a renormalizable framework and new fixed points of the renormalization-group equation.

\section{The magnetic moment of the $\rho$-meson}

In Ref. [1], we have analyzed the pion mass dependence of the magnetic moment of the $\rho$-meson at the leading one-

\footnotetext{
^e-mail: evgeny.epelbaum@rub.de

$\star \star$ e-mail: hermann.krebs@rub.de

$\star \star \star$ e-mail: meissner@hiskp.uni-bonn.de
}

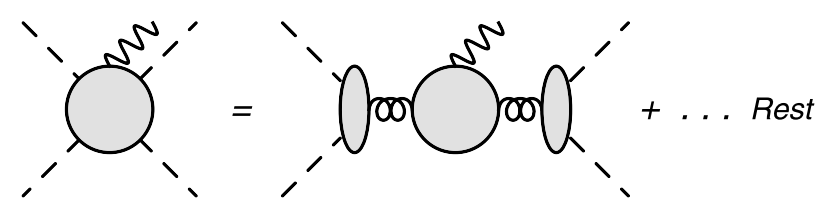

Figure 1. The reaction $\pi \pi \rightarrow \gamma \pi \pi$. Dashed, wavy and wiggled lines refer to pions, photons and $\rho$-mesons, respectively. "Rest" denotes the non-resonant contributions.

loop order. To this aim, we have employed the most general chiral effective Lagrangian for $\rho$ - and $\omega$-mesons, pions and external sources

$$
\mathcal{L}=\mathcal{L}_{\pi}+\mathcal{L}_{\rho \pi}+\mathcal{L}_{\omega}+\mathcal{L}_{\omega \rho \pi},
$$

where the individual terms are taken in the parametrization of the model III of Ref. [2]. For a general review of effective chiral Lagrangians with vector mesons, see Ref. [3]. We made use of the universality of the vector-meson couplings, which was shown in Ref. [4] to follow from the consistency of an effective field theory (EFT) with respect to perturbative renormalization. Furthermore, the coupling constant $g_{\rho \pi \pi}$ obeys the KSFR relation $M_{\rho}^{2}=2 g_{\rho \pi \pi}^{2} F_{\pi}^{2}$, with $F_{\pi}$ denoting the pion decay constant.

The magnetic moment of the $\rho$-meson can be extracted from the resonant part of the five-point function in the reaction $\pi \pi \rightarrow \gamma \pi \pi$ as visualized in Fig. 1. Assuming that the interaction terms with a higher number of derivatives and/or more fields are suppressed by powers of some large hadronic scale, we have performed a one-loop calculation in terms of the expansion parameter $\xi=g_{\rho \pi \pi}^{2} /(4 \pi)^{2} \simeq 0.2$. At tree level, the magnetic moment is found to be real, and the gyromagnetic ratio is $g_{\rho}=2$. One-loop diagrams yield corrections of the order of $10 \%$ and lead to a small imaginary part of $g_{\rho}$. The resulting pion mass dependence of $g_{\rho}$ is shown in Fig. 2. At the physical point, we found $\operatorname{Re}\left(g_{\rho}\right) \simeq 2.2$ in good agreement with the most recent lattice QCD calculation of Ref. [5], $\left(g_{\rho}\right)_{\text {unquenched }}=2.4(2)$. 


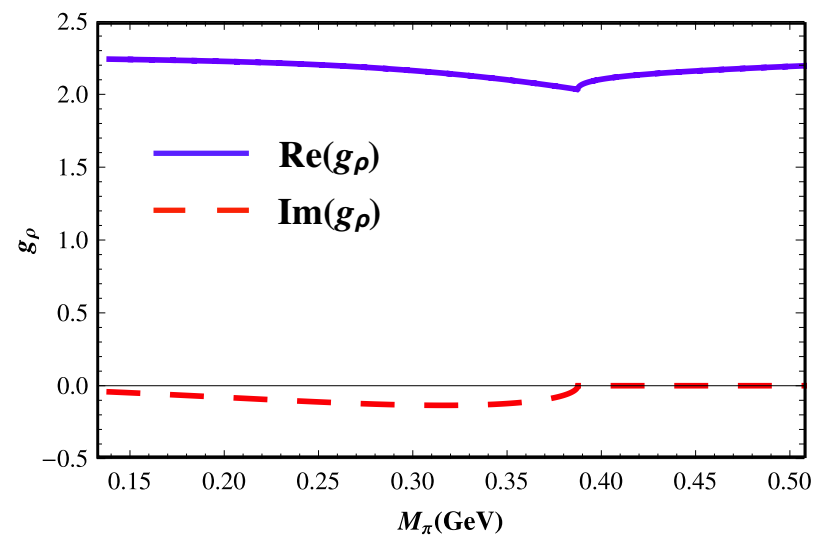

Figure 2. The gyromagnetic ratio $g_{\rho}$ of the $\rho$-meson as a function of the pion mass.

Earlier lattice QCD studies have reported the values of $\left(g_{\rho}\right)_{\text {quenched }} \sim 2.3[6],\left(g_{\rho}\right)_{\text {unquenched }}=1.6(1)[7]$.

\section{Elastic pion-nucleon scattering}

Elastic pion-nucleon $(\pi N)$ scattering certainly belongs to the the most extensively studied processes in ChPT [8-16]. The $\pi N$ scattering amplitude enters as a subprocess into the interactions between two and three nucleons at large distances. Thus, its detailed understanding is a necessary pre-requisite for deriving high-precision nuclear forces in chiral EFT [14, 17, 18].

In our paper [19], we have analyzed $\pi N$ scattering up to fourth order $Q^{4}$ within different formulations of ChPT. Specifically, we employed the heavy-baryon (HB) formulations utilizing the standard counting of the nucleon mass as $m_{N} \sim \Lambda_{b}(\mathrm{HB}-\pi \mathrm{N})$ with $\Lambda_{b}$ denoting the breakdown scale of the chiral expansion, and the counting scheme with $m_{N} \sim \Lambda_{b}^{2} / Q$ (HB-NN) employed in few-nucleon studies. We have also performed calculations within a covariant (Cov) formulation of ChPT based on an extended on-mass-shell (EOMS) renormalization scheme [20-22]. Our analysis differs in several aspects from the already mentioned earlier studied of this topic. First, the determination of the low-energy constants (LECs) was carried out using directly the available $\pi N$ scattering data instead of having to rely on partial wave analyses (PWA), see also Ref. [23] for a similar approach. Secondly, we have benefited from the developments in the project B.6 by employing a novel approach to estimate the theoretical uncertainty from the truncation of the chiral expansion formulated in Ref. [17] in order to stabilize the fits with respect to the energy range. Finally, we have discussed in detail the renormalization and finite shifts of the various LECs within the covariant formulations and studied the role of the $\Delta(1232)$ isobar by treating it as an explicit degree of freedom at lowest order in the chiral expansion.

The main results of our study can be summarized as follows. In the $\Delta$-less case, all three considered approaches are found to lead to a comparable description of lowenergy $\pi N$ data, and the extracted values of the LECs compare well with those reported in the literature at the cor-
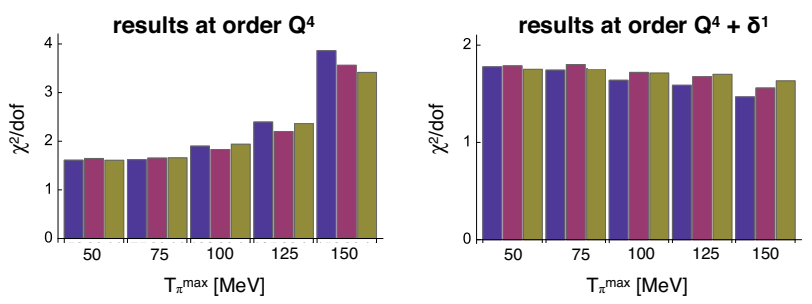

Figure 3. Reduced $\chi^{2}$ for fits up to various values of the maximal energy $T_{\pi}^{\max }$ in the $\Delta$-less (left panel) and $\Delta$-full (right panel) formulations of ChPT. The blue/red/green bars denote the results for the HB-NN/HB- $\pi \mathrm{N} / \mathrm{Cov}$ counting. The theoretical uncertainty is estimated using the approach of Ref. [17] and assuming $\Lambda_{b} \sim 600 \mathrm{MeV}$.

responding chiral orders. While the inclusion of the theoretical uncertainties in the definition of the $\chi^{2}$ helps to stabilize the fits by decreasing the relative weight of highenergy data, we found clear indications of the lower breakdown scale of the chiral expansion than the assumed one of $\Lambda_{b} \sim 600 \mathrm{MeV}$ [17]. This conclusion is supported by the increasing behavior of the reduced $\chi^{2}$ with energy as visualized in the left panel of Fig. 3. It is also reflected in the extracted values of the LECs, which show sizable sensitivity on the energy range used in the fits, see the upper panel of Fig. 4, and to the chiral order. Furthermore, some of the LECs take rather large numerical values. For example, at order $Q^{4}$ in the covariant approach, we found the values of

$$
c_{1} \simeq-0.82, \quad c_{2} \simeq-3.56, \quad c_{3} \simeq-4.59, \quad c_{4} \simeq 3.44
$$

for the order- $Q^{2}$ LECs in units of $\mathrm{GeV}^{-1}$ and

$$
d_{1+2} \simeq 5.43, \quad d_{3} \simeq-4.58, \quad d_{4} \simeq-0.40, d_{14-15} \simeq-9.94
$$

for the relevant order- $Q^{3} \mathrm{LECs}$ in units of $\mathrm{GeV}^{-2}$.

Clearly, one expects the convergence of the chiral expansion for $\pi N$ scattering to be slow due to the appearance of the $\Delta(1232)$ resonance. The situation can be improved by treating the $\Delta$-isobar as an explicit degree of freedom [24-26]. To quantify the effects of the $\Delta$ isobar, we have repeated our analysis including its lowestorder contributions. The only new parameters, namely the
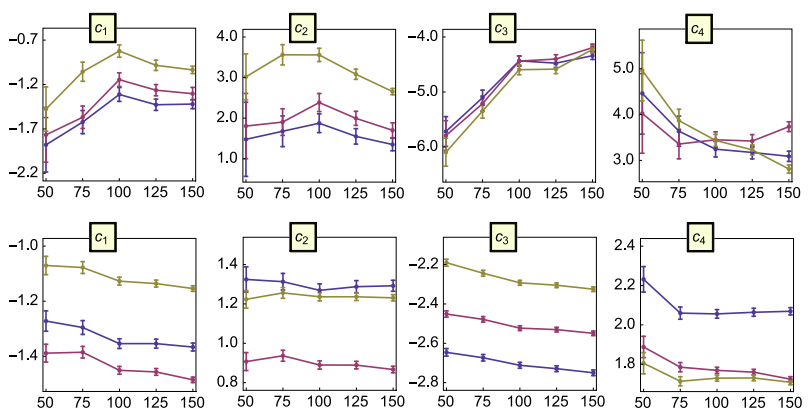

Figure 4. The dependence of the order- $Q^{2} \pi N$ LECs $c_{i}$, given in units of $\mathrm{GeV}^{-1}$, on the maximal incoming pion energy $T_{\pi}$ used in the fits at order $Q^{4}$ in the $\Delta$-less approach (upper panel) and at order $Q^{4}+\delta^{1}$ in the $\Delta$-full approach (lower panel). The blue/red/green points and lines denote the results of the HB$\mathrm{NN} / \mathrm{HB}-\pi \mathrm{N} / \mathrm{Cov}$ formulations. 

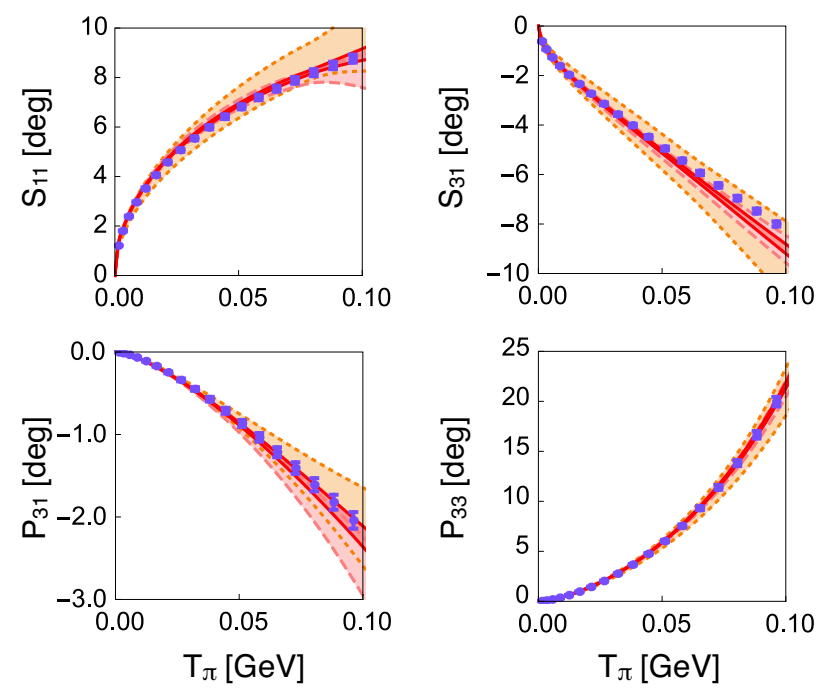

Figure 5. Predictions for selected S- and P-wave phase shifts up to $T_{\pi}=100 \mathrm{MeV}$ in the covariant $\Delta$-full formulation of chiral EFT. Orange dotted, pink dashed and red solid bands refer to $Q^{2}+\delta^{1}, Q^{3}+\delta^{1}$ and $Q^{4}+\delta^{1}$ results, respectively. The truncation errors are estimated using the approach of Ref. [17] and assuming $\Lambda_{b} \sim 600 \mathrm{MeV}$. Blue points show the results of the RS equation analysis of Ref. [27].

mass of the $\Delta$ and the $\pi N \Delta$ coupling constant, were fixed to its Breit-Wigner and the large- $N_{c}$ values, respectively: $m_{\Delta}=1.232 \mathrm{GeV}, g_{\pi N \Delta}=3 /(2 \sqrt{2}) g_{A}=1.35$. While the quality of the reproduction of the $\pi N$ scattering data well below the $\Delta$-region is not significantly impacted by the explicit inclusion of the $\Delta$-isobar, we indeed found an improved convergence of the resulting EFT. The corresponding breakdown scale is consistent with or even slightly larger than the estimated $\Lambda_{b} \sim 600 \mathrm{MeV}$, see the right panel of Fig. 3. Not surprisingly, the $\Delta$-full formulation allows for a more reliable determination of the LECs, which turn out to be rather stable with respect to choosing the fitting range, see Fig. 4. We also found all LECs in the $\Delta$ full approach to be of a natural size. In particular, at order $Q^{4}+\delta^{1}$ in the covariant approach, we obtained the values

$$
c_{1} \simeq-1.15, \quad c_{2} \simeq-1.57, \quad c_{3} \simeq-2.54, \quad c_{4} \simeq 2.61
$$

for the order- $Q^{2}$ LECs and

$$
d_{1+2} \simeq 1.27, \quad d_{3} \simeq-1.83, \quad d_{4} \simeq 0.37, d_{14-15} \simeq-2.22
$$

for the relevant order- $Q^{3} \pi N$ LECs, to be compared with the values in the $\Delta$-less approach listed above.

Last but not least, the resulting phase shifts are found to be in a very good agreement with the ones determined within the project B.3 in the framework of Roy-Steiner (RS) equations, where detailed error estimates of all input quantities, the solution procedure and truncations was performed [27-29]. As a representative example, we show in in Fig. 5 our predictions for selected S- and P-waves along with the estimated truncation errors in comparison with the results from Ref. [27].

While the above results look rather promising and indicate the importance of the explicit treatment of the $\Delta$ isobar, its inclusion beyond the tree level in the covariant
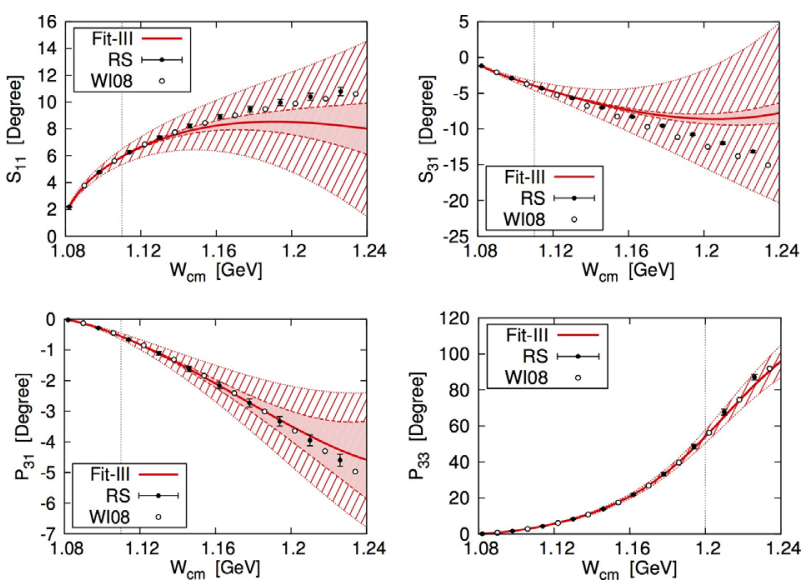

Figure 6. Phase shifts obtained from the $\Delta$-full covariant ChPT at leading one-loop order. Dots with error bars stand for the RS phase shifts [27] while circles without error bars represent the phase shifts from the George Washington partial wave analysis [32]. The solid (red) line represents the result of Fit III of Ref. [30]. The red narrow error bands correspond to the uncertainties propagated from the errors of LECs while the wide dashed error bands show truncation uncertainties at order $Q^{3}$ estimated using the approach of Ref. [17].

framework is a rather challenging task due to a large complexity of the calculations emerging from the $\Delta$ propagators. In Ref. [30], we have performed a complete analysis at the leading one-loop order (i.e. $Q^{3}$ ) in the covariant chiral EFT with explicit $\Delta$ degrees of freedom. In this work, all possible nucleon- and $\Delta$-propagator contributions have been calculated up to the leading one-loop order. To achieve this goal, we applied the EOMS (complexmass [31]) scheme to diagrams involving pion and nucleon lines (delta lines) and determined the corresponding renormalized LECs by performing fits to the phase shifts obtained in the RS equation analysis of Ref. [27] up to the center of mass energy of $1.11 \mathrm{GeV}\left(1.2 \mathrm{GeV}\right.$ for the $P_{33}$ partial wave). In Fig. 6, we show our results for the selected partial waves along with the theoretical uncertainties.

We have also looked at the $\pi N$ sigma term but were unable to obtain an accurate prediction for this quantity. Our results are, however, consistent with the value found in the RS analysis, $\sigma_{\pi N}=59.1 \pm 3.5 \mathrm{MeV}$ within the large errors of our determination. We have also extended these studies to the SU(3) sector by calculating the masses and the corresponding sigma terms of the baryon octet and studying the strangeness content of the nucleon.

\section{The reaction $\pi N \rightarrow \pi \pi N$}

In addition to the elastic channel of $\pi N$ scattering, we have studied in Ref. [33] single pion production off nucleons from threshold up to the delta resonance region, extending earlier ChPT studies from Refs. [34-37]. With three pions in the initial and final states, the theoretical study of this process provides complimentary information to elastic scattering and offers access to certain LECs which are not probed in the reaction $\pi N \rightarrow \pi N$. In particular, the 

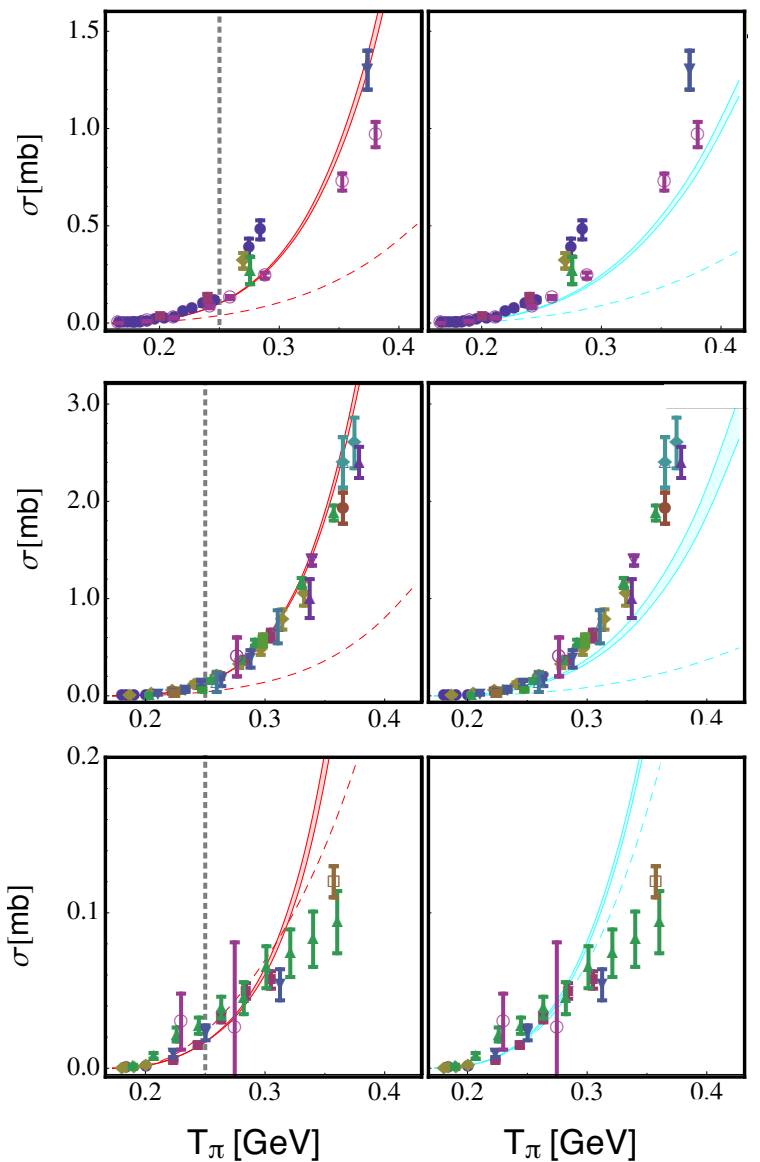

Figure 7. Predictions for the total cross section up to $T_{\pi} \simeq$ $400 \mathrm{MeV}$ in the covariant $\Delta$-full (left panel) and $\Delta$-less (right panel) versions of chiral EFT. Dashed and solid lines refer to LO and NLO results, respectively. The maximal energy used in the fit at NLO is marked by the vertical dotted line. The bands at NLO reflect the uncertainty from using different partial wave analyses in the determination of the LECs $c_{i}$ as explained in the text. Experimental data are from Refs. [45-47].

LEC $d_{16}$ is of great interest as it governs the quark mass dependence of the axial charge of the nucleon. If fact, the lack of knowledge of the precise value of $d_{16}$ is one of the main sources of theoretical uncertainty in chiral extrapolations of nuclear observables [38-44]. Notice further that a good theoretical understanding of this process is expected to provide useful constraints for multi-channel PWA of $\gamma$ and $\pi$-induced reactions off the nucleon, which is the main task of the project A.2.

In our exploratory study, we have restricted ourselves to calculating the leading (LO), i.e. $Q$, and subleadingorder (NLO), i.e. $Q^{2}$ contributions to the scattering amplitude which emerge from tree-level diagrams. We have used both the HB and Cov formulations of baryon chiral EFT with and without explicit $\Delta$ degrees of freedom. Using the large- $N_{c}$ value for the $\pi N \Delta$ coupling constant, the results at LO come as parameter free predictions. At NLO, several LECs have to be determined. For the LECs $c_{i}$ from $\mathcal{L}_{\pi N}^{(2)}$, we have adopted the values extracted from two different PWA of elastic $\pi N$ scattering. Thus, our $\Delta-$ less results at NLO are parameter free, too. In the HB
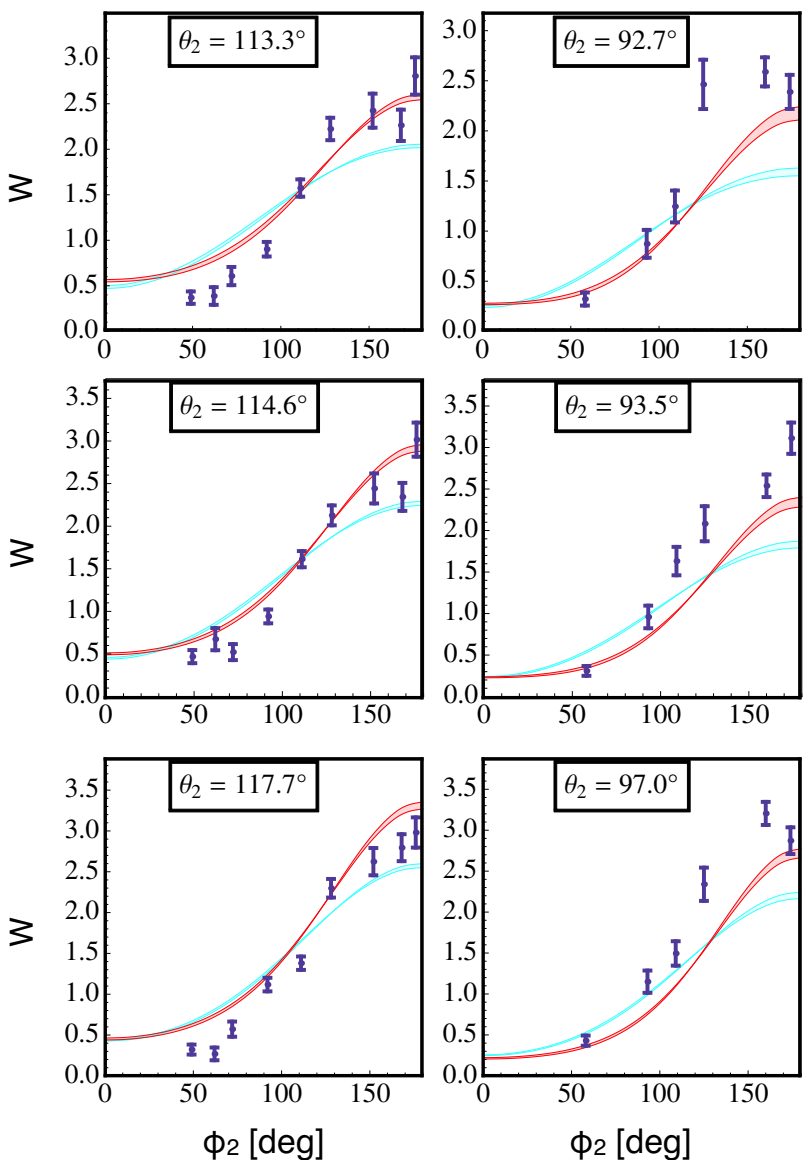

Figure 8. NLO predictions for the angular correlation functions in the $\pi^{-} p \rightarrow \pi^{+} \pi^{-} n$ channel at fixed values of $\theta_{2}$ and $\theta_{1}=91^{\circ}$ (upper panel), $\theta_{1}=76^{\circ}$ (middle panel) and $\theta_{1}=71^{\circ}$ (lower panel) for $\sqrt{s}=1301 \mathrm{MeV}$ in the $\Delta$-full (red lines) and $\Delta$-less (cyan lines) covariant chiral EFT approaches. The bands at NLO reflect the uncertainty from using different partial wave analyses in the determination of the LECs $c_{i}$ as explained in the text. Experimental data are from Ref. [48].

$\Delta$-full approach, one is left with three unknown LECs, namely $b_{4}, b_{5}$ from $\mathcal{L}_{\pi N \Delta}^{(2)}$ and $g_{1}$ from $\mathcal{L}_{\pi \Delta}^{(1)}$. In the covariant framework, one, in addition, encounters the contributions from the LECs $b_{3}$ and $b_{6}$. These LECs have been determined from the available total cross section data in the reactions $\pi^{-} p \rightarrow \pi^{0} \pi^{0} n, \pi^{-} p \rightarrow \pi^{+} \pi^{-} n, \pi^{+} p \rightarrow \pi^{+} \pi^{+} n$, $\pi^{+} p \rightarrow \pi^{+} \pi^{0} p$ and $\pi^{-} p \rightarrow \pi^{0} \pi^{-} p$, see Fig. 7 for representative examples. The extracted values of $g_{1}$ are found to be consistent with its large- $N_{c}$ value of $g_{1}=2.27$. We also found strong anticorrelations between the LECs $b_{4}$ and $b_{5}$ as well as $b_{3}$ and $b_{6}$, which prevented a reliable determination of the linear combinations.

With all LECs being determined as described above, we have looked at various differential observables. As a representative example, we show in Fig. 8 our predictions for the angular correlation function $W$, see Ref. [33] for a precise definition. Here $\theta_{1,2}$ denote the angles between the momentum of the incoming and the two outgoing pions, while $\phi_{2}$ is the azimuthal angle of one of the outgoing pi- 


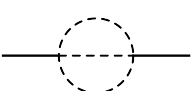

a)

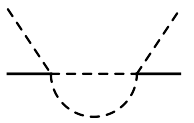

d)

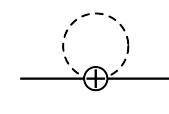

b)

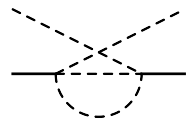

e)

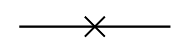

c)

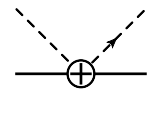

f)
Figure 9. Diagrams a)-c): The two-point function at the twoloop order. Solid and dashed lines refer to the heavy and light particles $\Psi$ and $\pi$, respectively. The vertices in graphs b) and c) denote one-loop- and two-loop-order counterterms. Diagrams d)-f): one-loop graphs contributing to $\Psi \pi$ scattering.

ons $^{1}$, see Ref. [33] for more details. Generally, the explicit treatment of the delta isobar is found to result in a better description of the data, most notably of the $\pi^{-} p \rightarrow \pi^{0} \pi^{0} n$ and $\pi^{-} p \rightarrow \pi^{+} \pi^{-} n$ total cross sections at higher energies and most of the differential cross sections. Still, certain single- and double-differential cross sections could not be properly described at this order in the chiral expansion. This calls for extending these calculations to next-higher order. Work along this line is in progress.

\section{New directions in chiral perturbation theory}

\subsection{Complex-mass renormalization scheme at the two-loop level}

ChPT can be straightforwardly extended to include meson and baryon resonances as dynamical degrees of freedom for soft processes, i.e. for reactions with external pion momenta and three-momenta of the nucleons of the order of $M_{\pi}$, see e.g. the already discussed applications of $\Delta$-full formulations of ChPT. On the other hand, formulating a consistent EFT at higher energy to study e.g. the properties of (axial-) vector mesons is a challenging task, see $[1,49,50]$ and references therein for the first steps along this line using the complex-mass renormalization scheme [31]. It is, however, not a priori clear that this scheme is applicable beyond the one-loop level, i.e. that the renormalized expressions fulfill the power counting.

To clarify this issue, we have studied in Ref. [51] the two-point function of a heavy scalar $\Psi$-meson, which can decay into three light scalar fields $\pi$, at the two-loop level, see diagrams (a-c) in Fig. 9. The restriction to a simple model has allowed us to avoid complications due to the spin and chiral structure of the EFT which are not relevant for the discussion of the applicability of the complexmass scheme. By explicitly calculating the diagrams a)c) in Fig. 9, with the one-loop counterterm being determined from $\pi \Psi$ scattering graphs d)-f), and by employing the complex-mass scheme, we were able to explicitly

\footnotetext{
${ }^{1}$ The coordinate frame is defined by choosing the $z$-axis in the direction of the incoming pion and letting one of the outgoing pions to lie in the $x z$-plane.
}
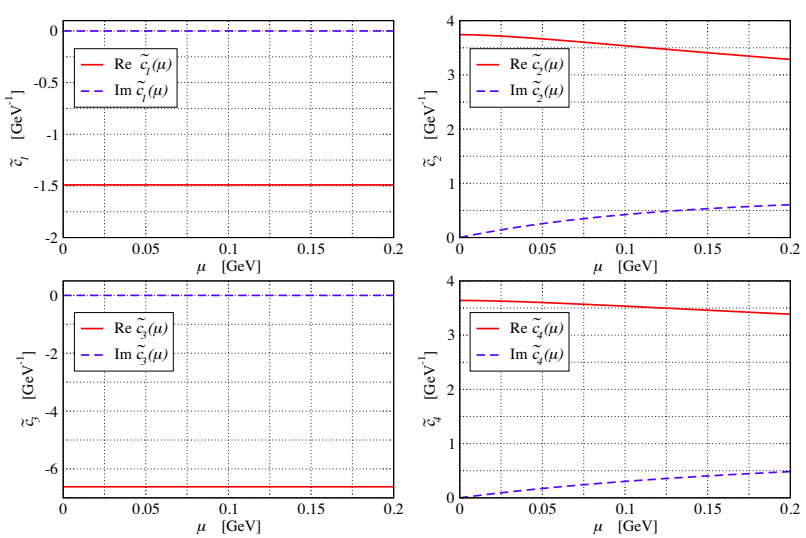

Figure 10. The renormalized LECs $c_{i}$ in the generalized EOMS scheme as functions of the sliding scale $\mu$.

show the absence of power-counting-breaking terms in the final renormalized expressions for the self-energy. Our work opens the way for a systematic analysis of e.g. the $\omega$-meson in chiral EFT.

\subsection{Baryon chiral perturbation theory beyond the low-energy region}

In Ref. [52], we have formulated a new approach to baryon ChPT which is applicable beyond the low-energy region at small scattering angles and applied it to the reaction $\pi N \rightarrow$ $\pi N$. Specifically, instead of considering the kinematical region of $t=O\left(Q^{2}\right)$ and $v \equiv(s-u) /\left(4 m_{N}\right)=O(Q)$ with $s, t$ and $u$ being the Mandelstam variables as it is usually the case in ChPT, we have demonstrated that the amplitude can be systematically calculated in the region of $t=O\left(Q^{2}\right)$ and $v^{2}-\mu^{2}=O(Q)$ even for $v=O(1)$. In this kinematical region, despite the higher energies, the quark structure of hadrons is still not resolved.

To achieve this goal, we have re-arranged the effective chiral Lagrangian according to a new power counting and exploited the freedom in the choice of renormalization conditions for loop diagrams. The re-arranged effective Lagrangian at any given order in the new counting contains a finite number of terms but involves some vertices of higher orders in the standard counting. Provided all the relevant resonances are treated as explicit degrees of freedom, the re-arranged effective Lagrangian generates a convergent Taylor series of the analytic part of the amplitude. Notice that the LECs of the re-arranged Lagrangian differ from the original ones by (known) $\mu$-dependent shifts.

To ensure that loop diagrams obey the new power counting, we have employed a generalization of the EOMS scheme by shifting the renormalization point into the physical region above the threshold. More precisely, the loop integrals are subtracted at the nucleon momentum $p^{2}=$ $m_{N}^{2}+2 m_{N} \mu$. Since this procedure results in subtracting also the imaginary parts of the integrals, the renormalized LECs become complex in our scheme for $\mu>0$ as visualized in Fig. 10. 

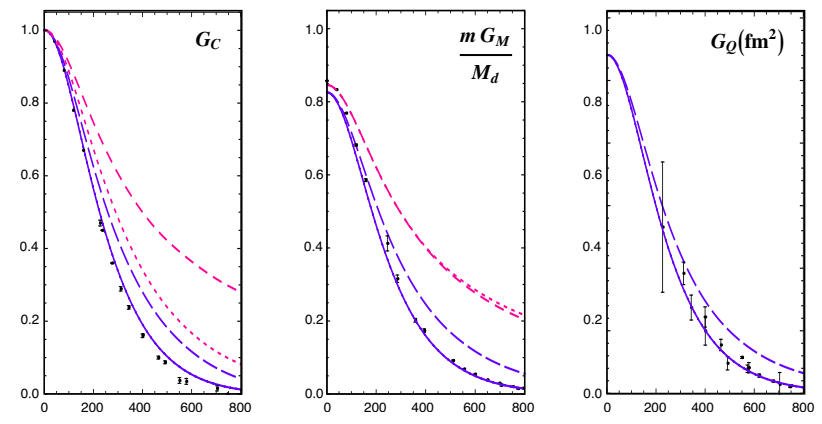

Figure 11. Electromagnetic form factors of the deuteron at LO in the renormalizable chiral EFT approach of Ref. [54] as a function of the momentum transfer $q$ in comparison with experimental data from Refs. [60, 61]. Solid and long-dashed violet lines (short- and medium-dashed pink lines) show the predictions in the chiral (pionless) EFT with and without using phenomenological form factors of the nucleon, respectively.

As an application, we have used the resulting framework to analyze $\pi N$ scattering at low energy. ${ }^{2}$ With the relevant LECs being fitted to the $\pi N$ PWA, all phase shifts in the threshold region were shown to be well described within the new formulation, with the results being similar to those discussed in section 4. Our new approach is not limited to elastic $\pi N$ scattering and is also applicable to pion photo- and electro-production reactions, Compton scattering and processes involving several pions.

\section{Applications in the two-nucleon sector}

\subsection{Deuteron electromagnetic form factors in a renormalizable formulation of chiral EFT}

In Ref. [53], we have analyzed the electromagnetic form factors $G_{C}, G_{M}$ and $G_{Q}$ of the deuteron at $\mathrm{LO}$ within a renormalizable formulation of chiral nuclear EFT proposed in Ref. [54], see Refs. [55-58] and references therein for related earlier studies. This approach is based on a manifestly Lorentz invariant form of the effective Lagrangian without performing a non-relativistic expansion for the nucleon propagators. The NN amplitude can then be calculated by solving the corresponding threedimensional integral equation which satisfies relativistic elastic unitarity [59]. The resulting scattering amplitude at LO in chiral EFT is renormalizable, which allows one to obtain regulator-independent results for observables.

At LO, the form factors of the deuteron only receive contributions from single-nucleon currents, and the results do not involve any free parameters. In Fig. 11, we show by the violet long-dashed lines the resulting LO parameter-free cutoff-independent predictions for $G_{C}, G_{M}$ and $G_{Q}$. Notice that the deviations from the experimental at momentum transfers of $q \sim 200 \mathrm{MeV}$ and higher can be largely traced back to the well-known slow convergence of the chiral expansion for the nucleon form factors $[62,63]$. Indeed, replacing the LO single-nucleon current

\footnotetext{
${ }^{2}$ To go beyond the threshold region, one would have to explicitly take into account the relevant contributions of the nucleon resonances. This task is postponed for future work.
}

by the dispersion-theoretical form factor parametrization of Ref. [64] as shown by the solid lines in Fig. 11, a very good description of the data is observed even at rather high values of $q$. The resulting predictions for the deuteron magnetic and quadrupole moments, $\mu^{\mathrm{LO}}=0.826(e /(2 m))$ and $Q^{\mathrm{LO}}=0.271 \mathrm{fm}$ are in a good agreement with the experimental values of $\mu^{\exp }=0.85741(e /(2 m))$ and $Q^{\exp }=$ $0.2859 \mathrm{fm}$.

The renormalizable approach also provides a clear evidence of the importance of the one-pion exchange NN potential by comparing the LO results in $\pi$-less and $\pi$-full versions of the EFT as visualized in Fig. 11. Notice that the quadrupole form factor of the deuteron vanishes at LO in the $\pi$-less approach.

\section{$6.2{ }^{1} \mathrm{~S}_{0} \mathrm{NN}$ scattering in the modified Weinberg approach}

While the LO calculations in the novel renormalizable approach to NN scattering of Ref. [54] look promising, the inclusion of higher-order corrections requires much more extensive calculations than in the standard, non-relativistic framework. In Ref. [65], we made a first step along this line by analyzing the subleading contact interaction in the ${ }^{1} \mathrm{~S}_{0}$ partial wave. Given that the singular tensor part of the one-pion exchange potential does not contribute in spinsinglet channels, it is possible to include the NLO contact interaction both perturbatively and without relying on perturbation theory. In the latter case, the scattering amplitude shows a residual dependence on the subtraction point $\mu$. We have argued that the unnaturally large value of the scattering length in the ${ }^{1} \mathrm{~S}_{0}$ channel requires the subtraction scale $\mu$ corresponding to the $\mathrm{LO}$ contact interaction to be chosen of the order of the hard scale in the problem. We have also addressed perturbativeness of the subleading contact interaction within our scheme and found it to be advantageous to treat it nonperturbatively at energies above $E_{\text {lab }} \sim 50 \mathrm{MeV}$. Finally, we have demonstrated that the predictions for the low-energy theorems for the coefficients in the effective range expansion are strongly improved when going from $\mathrm{LO}$ to NLO.

\subsection{New fixed points of the renormalization group for nonrelativistic two-body systems}

The renormalization group (RG) is known to be a powerful tool to study the scale dependence of systems in various areas of physics. For a system of two nonrelativistic particles interacting via short-range forces, the nontrivial fixed point describing a system in the "unitary limit" and analyzed in Ref. [66] has attracted much interest in the context of NN scattering in the ${ }^{1} \mathrm{~S}_{0}$ and ${ }^{3} \mathrm{~S}_{1}$ channels. While the existence of other nontrivial fixed points of the RG equation for two-body scattering has been suggested in the literature, no explicit results were known. In Ref. [67], we have presented a general method for constructing an infinite number of possible fixed points by utilizing a separable ansatz for the potentials that arise in EFT description 
of such systems. Our results indicate a much richer structure than previously recognized in the RG flows of simple short-range potentials.

We would like to thank our collaborators Véronique Bernard, Michael Birse, Dalibor Djukanovic, Ashot Gasparyan, Jambul Gegelia, Matthias Schindler, Dmitrij Siemens, and De-Liang Yao. The work reported here would not have been possible without the support from the Deutsche Forschungsgemeinschaft within the SFB/TR16.

\section{References}

[1] D. Djukanovic, E. Epelbaum, J. Gegelia and U.-G. Meißner, Phys. Lett. B 730, 115 (2014) [arXiv:1309.3991 [hep-ph]].

[2] G. Ecker, J. Gasser, H. Leutwyler, A. Pich and E. de Rafael, Phys. Lett. B 223, 425 (1989).

[3] U.-G. Meisßner, Phys. Rept. 161, 213 (1988).

[4] D. Djukanovic, M. R. Schindler, J. Gegelia, G. Japaridze and S. Scherer, Phys. Rev. Lett. 93, 122002 (2004) [hep-ph/0407239].

[5] E. V. Luschevskaya, O. A. Kochetkov, O. V. Teryaev and O. E. Solovjeva, JETP Lett. 101, no. 10, 674 (2015).

[6] J. N. Hedditch et al., Phys. Rev. D 75, 094504 (2007) [hep-lat/0703014 [HEP-LAT]].

[7] M. Gurtler et al. [QCDSF Collaboration], PoS LATTICE 2008, 051 (2008).

[8] N. Fettes, U.-G. Meißner and S. Steininger, Nucl. Phys. A 640, 199 (1998) [hep-ph/9803266].

[9] P. Buettiker and U.-G. Meißner, Nucl. Phys. A 668, 97 (2000) [hep-ph/9908247].

[10] N. Fettes and U.-G. Meißner, Nucl. Phys. A 676, 311 (2000) [hep-ph/0002162].

[11] T. Becher and H. Leutwyler, JHEP 0106, 017 (2001) [hep-ph/0103263].

[12] M. Hoferichter, B. Kubis and U.-G. Meißner, Nucl. Phys. A 833, 18 (2010) [arXiv:0909.4390 [hep-ph]].

[13] J. M. Alarcon, J. Martin Camalich and J. A. Oller, Phys. Rev. D 85, 051503 (2012) [arXiv:1110.3797 [hep-ph]].

[14] H. Krebs, A. Gasparyan and E. Epelbaum, Phys. Rev. C 85, 054006 (2012) [arXiv:1203.0067 [nucl-th]].

[15] J. M. Alarcon, J. Martin Camalich and J. A. Oller, Annals Phys. 336, 413 (2013) [arXiv:1210.4450 [hep$\mathrm{ph}]$.

[16] Y. H. Chen, D. L. Yao and H. Q. Zheng, Phys. Rev. D 87, 054019 (2013) [arXiv:1212.1893 [hep-ph]].

[17] E. Epelbaum, H. Krebs and U.-G. Meißner, Eur. Phys. J. A 51, no. 5, 53 (2015) [arXiv:1412.0142 [nuclth]].

[18] E. Epelbaum, H. Krebs and U.-G. Meißner, Phys. Rev. Lett. 115, no. 12, 122301 (2015) [arXiv:1412.4623 [nucl-th]].

[19] D. Siemens, V. Bernard, E. Epelbaum, A. Gasparyan, H. Krebs and U.-G. Meißner, Phys. Rev. C 94, no.1, 014620 (2016) [arXiv:1602.02640 [nucl-th]].
[20] J. Gegelia and G. Japaridze, Phys. Rev. D 60, 114038 (1999) [hep-ph/9908377].

[21] J. Gegelia, G. Japaridze and X. Q. Wang, J. Phys. G 29, 2303 (2003) [hep-ph/9910260].

[22] T. Fuchs, J. Gegelia, G. Japaridze and S. Scherer, Phys. Rev. D 68, 056005 (2003) [hep-ph/0302117].

[23] K. A. Wendt, B. D. Carlsson and A. Ekström, arXiv:1410.0646 [nucl-th].

[24] E. E. Jenkins and A. V. Manohar, Phys. Lett. B 259, 353 (1991).

[25] T. R. Hemmert, B. R. Holstein and J. Kambor, J. Phys. G 24, 1831 (1998) [hep-ph/9712496].

[26] V. Pascalutsa and D. R. Phillips, Phys. Rev. C 67, 055202 (2003) [nucl-th/0212024].

[27] M. Hoferichter, J. Ruiz de Elvira, B. Kubis and U.-G. Meißner, Phys. Rev. Lett. 115, 092301 (2015) [arXiv:1506.04142 [hep-ph]].

[28] M. Hoferichter, J. Ruiz de Elvira, B. Kubis and U.-G. Meißner, Phys. Rev. Lett. 115, no. 19, 192301 (2015) [arXiv:1507.07552 [nucl-th]].

[29] M. Hoferichter, J. Ruiz de Elvira, B. Kubis and U.-G. Meißner, Phys. Rept. 625, 1 (2016) [arXiv:1510.06039 [hep-ph]].

[30] D. L. Yao, D. Siemens, V. Bernard, E. Epelbaum, A. M. Gasparyan, J. Gegelia, H. Krebs and U.-G. Meißner, JHEP 1605, 038 (2016) [arXiv:1603.03638 [hep-ph]].

[31] A. Denner, S. Dittmaier, M. Roth and D. Wackeroth, Nucl. Phys. B 560, 33 (1999) [hep-ph/9904472].

[32] R. A. Arndt, W. J. Briscoe, I. I. Strakovsky and R. L. Workman, Phys. Rev. C 74, 045205 (2006) [nuclth/0605082].

[33] D. Siemens, V. Bernard, E. Epelbaum, H. Krebs and U.-G. Meißner, Phys. Rev. C 89, no. 6, 065211 (2014) [arXiv:1403.2510 [nucl-th]].

[34] V. Bernard, N. Kaiser and U.-G. Meißner, Phys. Lett. B 332, 415 (1994) Erratum: [Phys. Lett. B 338, 520 (1994)] [hep-ph/9404236].

[35] V. Bernard, N. Kaiser and U.-G. Meißner, Nucl. Phys. B 457, 147 (1995) [hep-ph/9507418].

[36] V. Bernard, N. Kaiser and U.-G. Meißner, Nucl. Phys. A 619, 261 (1997) [hep-ph/9703218].

[37] N. Fettes, V. Bernard and U.-G. Meißner, Nucl. Phys. A 669, 269 (2000) [hep-ph/9907276].

[38] E. Epelbaum, U.-G. Meißner and W. Glöckle, Nucl. Phys. A 714, 535 (2003) [nucl-th/0207089].

[39] E. Epelbaum, U.-G. Meißner and W. Glöckle, nuclth/0208040.

[40] S. R. Beane and M. J. Savage, Nucl. Phys. A 713, 148 (2003) [hep-ph/0206113].

[41] S. R. Beane and M. J. Savage, Nucl. Phys. A 717, 91 (2003) [nucl-th/0208021].

[42] J. C. Berengut, E. Epelbaum, V. V. Flambaum, C. Hanhart, U.-G. Meißner, J. Nebreda and J. R. Pelaez, Phys. Rev. D 87, no. 8, 085018 (2013) [arXiv:1301.1738 [nucl-th]]. 
[43] E. Epelbaum, H. Krebs, T. A. Lähde, D. Lee and U.-G. Meißner, Phys. Rev. Lett. 110, no. 11, 112502 (2013) [arXiv:1212.4181 [nucl-th]].

[44] E. Epelbaum, H. Krebs, T. A. Lähde, D. Lee and U.-G. Meißner, Eur. Phys. J. A 49, 82 (2013) [arXiv:1303.4856 [nucl-th]].

[45] V. V. Vereshagin et al., Nucl. Phys. A 592, 413 (1995) [hep-ph/9504361].

[46] M. Kermani et al. [CHAOS Collaboration], Phys. Rev. C 58, 3419 (1998).

[47] J. B. Lange et al., Phys. Rev. Lett. 80, 1597 (1998).

[48] R. Muller et al., Phys. Rev. C 48, 981 (1993).

[49] D. Djukanovic, J. Gegelia and S. Scherer, Phys. Lett. B 690, 123 (2010) [arXiv:0903.0736 [hep-ph]].

[50] T. Bauer, J. Gegelia and S. Scherer, Phys. Lett. B 715, 234 (2012) [arXiv:1208.2598 [hep-ph]].

[51] D. Djukanovic, E. Epelbaum, J. Gegelia, H. Krebs and U.-G. Meißner, Eur. Phys. J. A 51, 101 (2015) [arXiv:1507.06771 [hep-ph]].

[52] E. Epelbaum, J. Gegelia, U.-G. Meißner and D. L. Yao, Eur. Phys. J. C 75, no. 10, 499 (2015) [arXiv:1510.02388 [hep-ph]].

[53] E. Epelbaum, A. M. Gasparyan, J. Gegelia and M. R. Schindler, Eur. Phys. J. A 50, 51 (2014) [arXiv:1311.7164 [nucl-th]].

[54] E. Epelbaum and J. Gegelia, Phys. Lett. B 716, 338 (2012) [arXiv:1207.2420 [nucl-th]].
[55] M. Walzl and U.-G. Meißner, Phys. Lett. B 513, 37 (2001) [nucl-th/0103020].

[56] D. R. Phillips, J. Phys. G 34, 365 (2007) [nuclth/0608036].

[57] M. P. Valderrama, A. Nogga, E. Ruiz Arriola and D. R. Phillips, Eur. Phys. J. A 36, 315 (2008) [arXiv:0711.4785 [nucl-th]].

[58] S. Kölling, E. Epelbaum and D. R. Phillips, Phys. Rev. C 86, 047001 (2012) [arXiv:1209.0837 [nucl-th]].

[59] V. G. Kadyshevsky, Nucl. Phys. B 6, 125 (1968).

[60] D. Abbott et al. [JLAB t20 Collaboration], Eur. Phys. J. A 7, 421 (2000) [nucl-ex/0002003].

[61] D. M. Nikolenko et al., Phys. Rev. Lett. 90, 072501 (2003).

[62] B. Kubis and U.-G. Meißner, Nucl. Phys. A 679, 698 (2001) [hep-ph/0007056].

[63] T. Bauer, J. C. Bernauer and S. Scherer, Phys. Rev. C 86, 065206 (2012) [arXiv:1209.3872 [nucl-th]].

[64] M. A. Belushkin, H.-W. Hammer and U.-G. Meißner, Phys. Rev. C 75, 035202 (2007) [hep-ph/0608337].

[65] E. Epelbaum, A. M. Gasparyan, J. Gegelia and H. Krebs, Eur. Phys. J. A 51, no. 6, 71 (2015) [arXiv:1501.01191 [nucl-th]].

[66] M. C. Birse, J. A. McGovern and K. G. Richardson, Phys. Lett. B 464, 169 (1999) [hep-ph/9807302].

[67] M. C. Birse, E. Epelbaum and J. Gegelia, Eur. Phys. J. A 52, no. 2, 26 (2016) [arXiv:1510.02209 [hep-ph]]. 